\title{
CONSTRUCTING FRAMED 4-MANIFOLDS WITH GIVEN ALMOST FRAMED BOUNDARIES
}

BY

\author{
STEVE J. KAPLAN
}

\begin{abstract}
Two methods are presented for constructing framed 4-manifolds with given almost framed boundaries. The main tools are the "moves" of Kirby's calculus of framed links. A new description is given for the $\mu$-invariant of a knot and this description is used to study almost framed 3-manifolds.
\end{abstract}

0. Introduction. The purpose of this paper is to study the types of framed 4-manifolds that a given closed, oriented 3-manifold bounds and to provide means of constructing framed 4-manifolds with a given boundary. There are six sections. $\S \S 1$ and 2 are concerned with preliminaries. The main result of $\$ 3$ is a constructive proof of the following theorem of Milnor [8]:

TheOREM 3.1 (MILNOR). Every closed, almost framed 3-manifold bounds a compact framed 4-manifold.

Our point of view is to regard the given 3-manifold as the boundary of a handlebody with a zero handle and all other handles of index 2 . The framed manifolds constructed can be pictured explicitly as handlebodies of this type. Our main tools are the two "moves" of Kirby's calculus of framed links [6]. As the proof involves several choices, many different framed 4-manifolds with a particular boundary can be generated. In order to illustrate the constructive aspect of the proof of Theorem 3.1, we show how to find an explicit handlebody presentation, with no handles of index 1 or 3 , for a closed almost parallelizable 4-manifold with index $=16$ and second betti number $=22$ (Theorem 3.3). This 4-manifold is homotopy equivalent to the Kummer surface [3], an example of interest to both topologists and algebraic geometers (see Remark 3.4).

The principal result of $\S 4$ (Theorem 4.2) is a second method for finding a framed 4-manifold with given boundary. In general, these 4-manifolds have smaller index and second betti number than those constructed in $\$ 3$, but are more difficult to produce explicitly. Formulas are provided allowing the

Received by the editors April 29, 1976 and, in revised form, February 11, 1977 and February 16, 1978.

AMS (MOS) subject classifications (1970). Primary 57A15; Secondary 55A25, 57A10.

Key words and phrases. Framed 4-manifold, calculus of framed links, knots. 
computation of the index and second betti number of these 4-manifolds without actually finding the manifolds themselves.

In order to utilize the formulas of Theorem 4.2, it is necessary to evaluate the $Z_{2}$ knot invariant, known as the $\mu$-invariant, first defined in Robertello [9] (see Definition 1.12). We give a new description of this invariant in $\$ 5$. It is shown that every knot in $S^{3}$ bounds a certain type of immersed disk (Theorem 5.2) called a singular ribbon (Definition 5.1). The $\mu$-invariant of the boundary of a singular ribbon can be evaluated by simply counting its triple points (Theorem 5.3).

In the final section, singular ribbons are employed to study links in $S^{\mathbf{3}}$ in which the linking number of any two components is zero. Specifically, it is shown (Theorem 6.1) that the components of such a link bound singular ribbons whose connected sums are again singular ribbons. Invariants of such a link relating to the $\mu$-invariants of connected sums of components of the link are defined. These invariants are also related to the homology intersection form of a 3-manifold associated to the link. By drawing on this dual relationship we derive the following result:

Theorem 6.12. Suppose $M$ is a closed, connected, oriented 3-manifold which bounds a framed manifold of index $k$. Suppose further that there exist elements $x, y, z \in H^{1}(M)$ such that $x \cup y \cup z$ is an odd multiple of the generator of $H^{3}(M)$. Then $M$ bounds a framed manifold of index $k+8$.

The machinery of $\$ 6$ is used together with the Kervaire-Milnor Theorem [5] to deduce the existence of classes in many bounded 4-manifolds which cannot be represented by smoothly embedded 2-spheres (Theorem 6.13).

$\S 1$ is devoted to preliminaries and many notational conventions set there are used throughout the paper. Examples are provided throughout to help the reader understand the workings of the constructions and invariants discussed.

The author wishes to express his deepest gratitude to Professor Robion C. Kirby for all of his help, both before and during the writing of this paper.

1. Preliminaries. In this section we explain the basic point of view of the paper and develop tools which will be needed later. Most of that which is contained herein is elementary and appears elsewhere. Many of the proofs are omitted.

Definition 1.1. A framed knot in $S^{3}$ is an embedded circle together with a distinguished homotopy class of sections of the normal circle bundle. A push-off in the framing is a representative of the homotopy class. A framed link in $S^{3}$ is a finite collection of disjoint framed knots.

REMARK 1.2. The framed knots corresponding to a particular embedding of $S^{1}$ in $S^{3}$ are in one-to-one correspondence with the integers. If $K$ is a component of a framed link $L$, we define $\operatorname{fr}(K)=\mathrm{lk}(K$, push-off $)$. 
To each framed link in $S^{3}$ is associated a compact, simply connected 4-manifold and a closed oriented 3-manifold. Regard $S^{3}$ as $\partial B^{4}$.

Definition 1.3. Suppose $L$ is a framed link in $S^{3}$. The 4-manifold associated to $L$, denoted by $W_{L}$, is built by attaching a 2-handle to $\partial B^{4}$ along each component of $L$ with the given framing. The 3-manifold associated to $L$, denoted by $M_{L}$, is the boundary of $W_{L}$.

REMARK 1.4. According to Lickorish [7] every closed connected, oriented 3-manifold is $M_{L}$ for some framed link $L$. The 4-manifolds associated to framed links are a good deal more tractable than general compact, 1-connected 4-manifolds and much of our later discussion will center on 4-manifolds of this type.

Definition 1.5. Suppose $L$ is a framed link with components $K_{1}, \ldots, K_{n}$. The linking matrix of $L$ is an $n \times n$ matrix of integers $a_{i j}$ whose entries are:

$$
\begin{aligned}
a_{i j} & =\operatorname{lk}\left(K_{i}, K_{j}\right) \quad \text { if } i \neq j, \\
& =\operatorname{fr}(K) \quad \text { if } i=j .
\end{aligned}
$$

REMARK 1.6. To each component $K$ of $L$ there corresponds an element $[K] \in H_{2}\left(W_{L} ; Z\right)$ which is represented by the union of the core of the handle over $K$ and the cone on $K$ in $B^{4}$. The group $H_{2}\left(W_{L} ; Z_{2}\right)$ is free abelian and the classes $\left[K_{i}\right]$ form a basis. The matrix associated to the bilinear form on $H_{2}\left(W_{L} ; Z\right)$ is the linking matrix of $L$.

Definition 1.7. A framing for a manifold $M$ is a distinguished trivialization of the tangent bundle $I(M)$. An almost framing for $M$ is a distinguished trivialization of the tangent bundle $T(M$-point).

REMARK 1.8. If $M^{3}$ is a closed, oriented 3-manifold, the almost framings for $M^{3}$ are in one-to-one correspondence with the elements of $H^{1}\left(M^{3} ; Z_{2}\right)$.

The main result of $\S 3$ will be that every closed, almost framed 3-manifold bounds a framed 4-manifold. The strategy will be to regard the given 3-manifold as $M_{L}$ for some framed link $L$ and then to modify $L$ so that the resulting $W_{L}$ can be framed. The most important tools will be the "moves" described in the following theorem by Kirby [6].

THEOREM (KIRBY). Suppose $M_{L_{1}}=M_{L_{2}}$ for framed links $L_{1}$ and $L_{2}$. Then $L_{1}$ can be transformed into $L_{2}$ by a sequence of moves of the following two types:

Move 1. Add to or delete from $L$ an unknotted component with framing \pm 1 which is separated from the other components by a 2-sphere.

Move 2. Replace a component of $L$ by its connected sum with a push-off of another component along some path in $S^{3}$.

REMARK 1.9. Move 1 replaces $W_{L}$ by $W_{L} \# \pm \mathbf{C} P^{2}$ when a component is added and splits off such a connected summand when one is deleted. Move 2 corresponds to changing the handlebody presentation by sliding one handle over another. The framing for the new component is found by considering the 
associated change of basis in $H_{2}\left(W_{L} ; Z\right)$ (see Remark 1.6). (For further discussion of Moves 1 and 2 see Kirby [6].)

Suppose $L$ is a framed link and $M_{L}$ is almost framed. Since $W_{L}$ is simply connected, the second Stiefel-Whitney class $w_{2}\left(W_{L}, M_{L}\right)$ is the sole obstruction to extending the given almost framing on $M_{L}$ to a framing on $W_{L}$.

Definition 1.10. A sublink $L^{1}$ of a framed link $L$ is characteristic (for the given almost framing on $M_{L}$ ) if the $\bmod 2$ reduction of $\left[L^{1}\right]$ is dual to $w_{2}\left(W_{2}, M_{L}\right)$.

REMARK 1.11. A sublink $L^{1}$ of $L$ is characteristic for some almost framing of $M_{L}$ if and only if $\left[L^{1}\right] \cdot x=x \cdot x \bmod 2$ for each $x \in H_{2}\left(W_{L} ; Z\right)$. In terms of the linking matrix $\left(a_{i j}\right)$ of $L$ this condition may be stated as

$$
\sum_{K_{i} \in L^{1}} a_{i j}=a_{i j} \bmod 2 \text { for each } 1<j<n .
$$

To each almost framing of $M_{L}$ corresponds a unique charactersitic sublink of $L$.

In $\S 4$, it will be necessary to consider the $Z_{2}$-valued knot invariant defined by Robertello [9]. A new description of this invariant is the main result of $\$ 5$.

Suppose $K \subset S^{3}$ is a knot. Choose a Seifert surface $F$ for $K$ and canonical curves $a_{1}, b_{1}, \ldots, a_{n}, b_{n}$ as a basis for $H_{1}(F)$. Define $\alpha_{i}$ (resp. $\beta_{i}$ ) to be the linking number of $a_{i}$ (resp. $b_{i}$ ) with its push-off along a normal vector field to $F$.

Definition 1.12. The invariant $\mu(K)$ is the mod 2 reduction of $\sum_{i=1}^{n} \alpha_{i} \beta_{i}$.

REMARK 1.13. If $\mu(K)=0$, it is possible to choose canonical curves so that each $\alpha_{i}(1<i<n)$ is zero $\bmod 2$ (see [2]).

2. Lemmas. The main tools which will be used in $\$ 3$ to construct framed manifolds with given boundaries are Kirby's Moves 1 and 2 (see \$1). In this section we derive several useful combinations of the moves. Since the effect these combinations have on framings varies according to the situations in which they are applied, the framings are suppressed. In any application they can be easily computed (Remark 1.9).

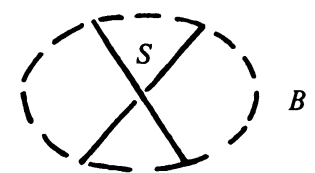

Case 1

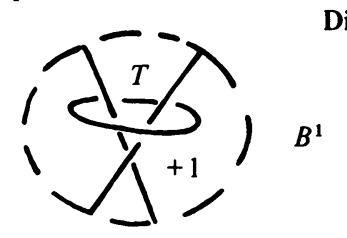

Diagram 2.1

Diagram 2.2

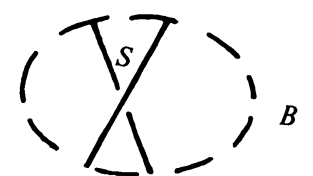

Case 2

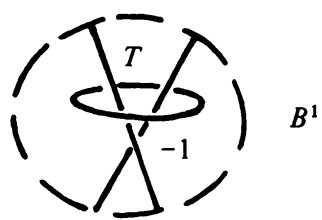


LEMMA 2.1. Suppose $L$ is a framed link and $B \subset S^{3}$ is a 3-ball intersecting $L$ in the complex $S$ shown in Diagram 2.1. Then $(B, S)$ may be replaced by the pair $\left(B^{1}, T\right)$ shown in Diagram 2.2 without changing $M_{L}$.

Proof. The proof is a sequence of Moves 1 and 2. We give the proof for the case on the left. The other case is analogous.
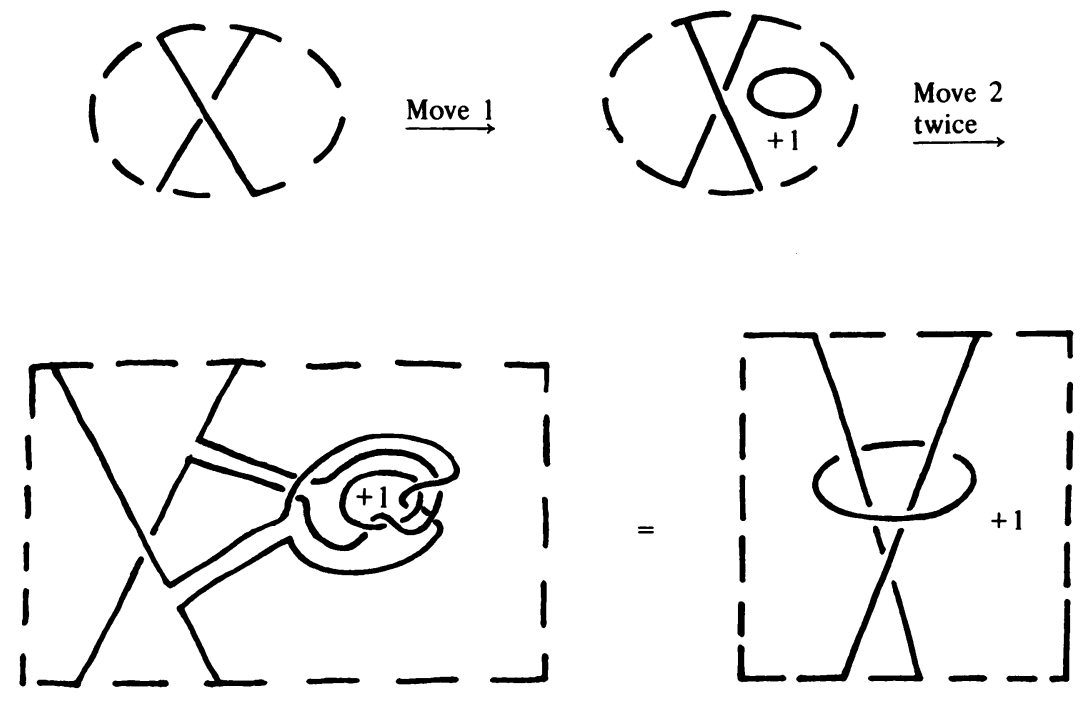

LEMMA 2.2. Suppose $L$ is a framed link and $B \subset S^{3}$ is a 3-ball intersecting $L$ in the complex $S$ shown in Diagram 2.3. Then $(B, S)$ may be replaced by the pair $\left(B^{1}, T\right)$ shown in Diagram 2.4 without changing $M_{L}$.
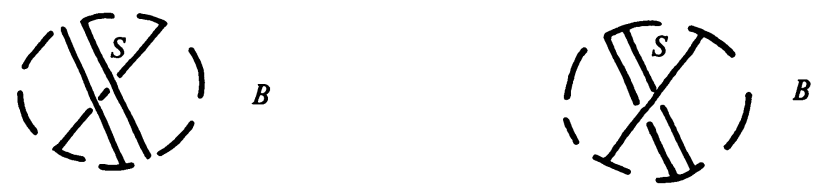

Diagram 2.3

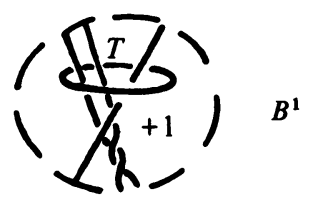

Diagram 2.4

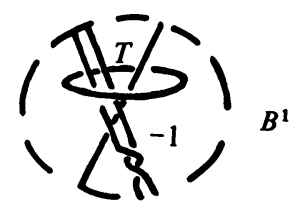


Proof. Again the proof is a sequence of Moves 1 and 2 and is given only in the case on the left.

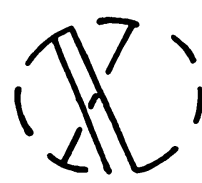

$\stackrel{\text { Move } 1}{\longrightarrow}$

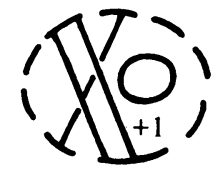

$\stackrel{\text { three times }}{\longrightarrow}$
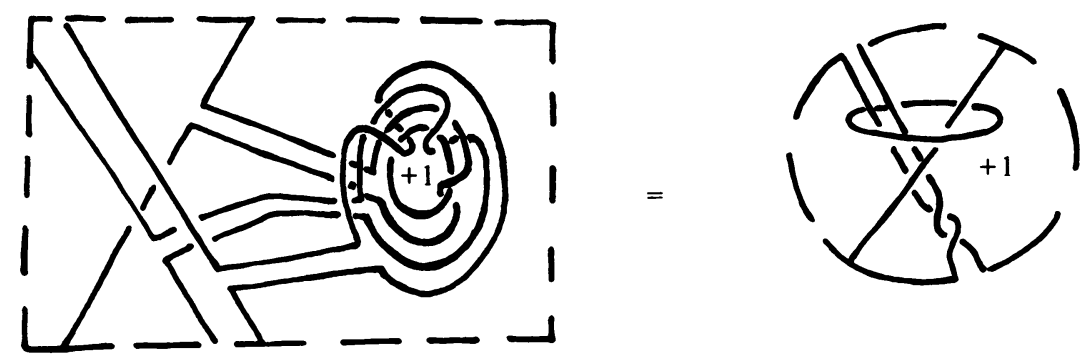

LEMMA 2.3. Suppose $L$ is a framed link and $B \subset S^{3}$ is a 3-ball intersecting $L$ in the complex $S$ shown in Diagram 2.5. Then $(B, S)$ may be replaced by the pair $\left(B^{1}, T\right)$ shown in Diagram 2.6 without changing $M_{L}$.
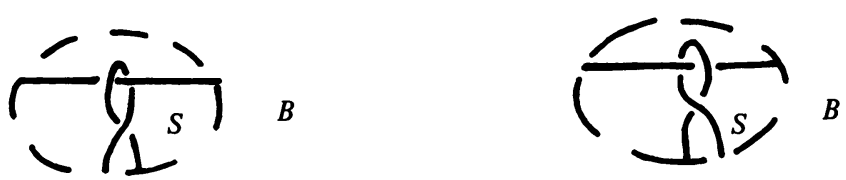

Case 1

Diagram 2.5

Case 2
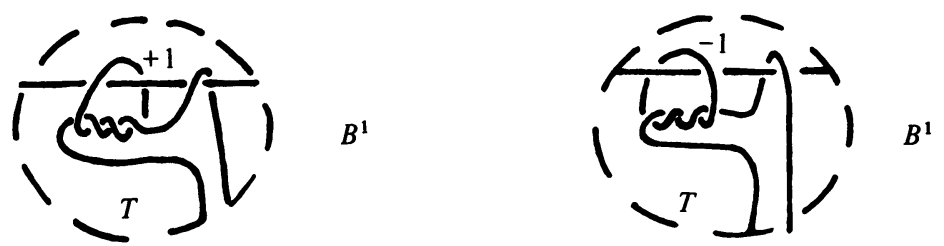

Diagram 2.6

Proof. The proof is a sequence of Moves 1 and 2. It is supplied only for the case on the left as the other is completely analogous. 


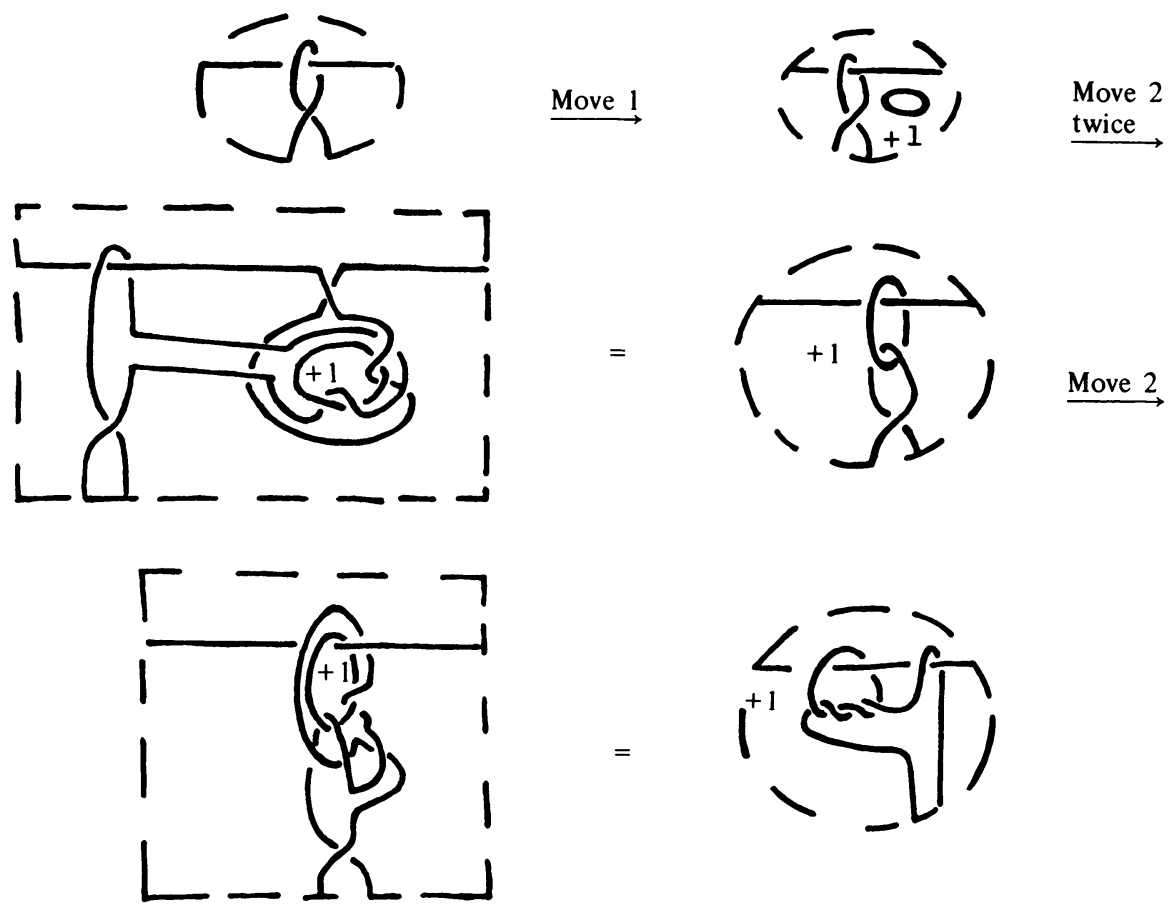

3. Constructing framed manifolds. The purpose of this section is to give an algorithmic proof of the following theorem of Milnor [8]:

THEOREM 3.1 (MILNOR). Every closed, almost framed 3-manifold bounds a compact, framed 4-manifold.

The main tools for the proof are Kirby's moves (see \$1) and the combinations of these moves derived in $\$ 2$. The proof will involve the consideration of several cases of increasing complexity. As an illustration of how the method of the proof can be used to construct framed 4-manifolds, the section concludes by showing how to find a handle presentation with no handles of index 1 or 3 for a closed almost parallelizable 4-manifold with index $=16$ and $\beta_{2}=22$.

Proof of THEOREM 3.1. It suffices to consider the case where $M^{3}$ is connected. Suppose $M^{3}$ is a closed, connected, almost framed 3-manifold. According to Lickorish [7], $M^{3}=M_{L}$ for some framed link $L$. Let $L^{1}$ be the characteristic sublink for the given almost framing.

Case $1 . L^{1}$ consists of a single unknotted component $K$ with framing \pm 1 .

If $K$ can be separated from the rest of $L$ by a 2-sphere, an application of Move 1 kills the characteristic class and the desired 4-manifold is the result. If 
$K$ cannot be separated from the rest of $L$ by a 2-sphere, let $D^{2}$ be a 2-disk bounded by $K$ which intersects each of the other components of $L$ transversely. Diagram 3.1 shows how to use Move 2 to remove a point of $L \cap\left(\right.$ int $D^{2}$ ).
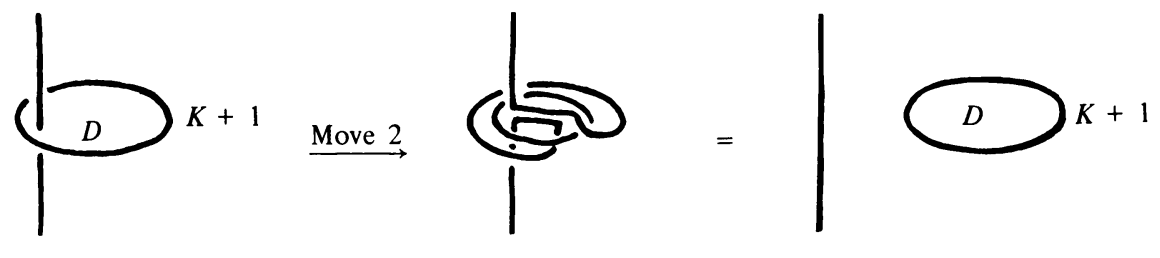

Diagram 3.1

After several applications of this procedure, it can be assumed that $L \cap$ (int $D^{2}$ ) $=\varnothing$. The boundary of a small regular neighborhood of $D^{2}$ is, then, a 2-sphere separating $K$ from the rest of $L$.

Case 2. $L^{1}$ consists of a single unknotted component $K$.

To reduce Case 2 to Case 1 , it is sufficient to use the moves to replace $L$ by a framed link in which a trivial knot with framing $[K] \cdot[K] \pm 1$ is characteristic. Use Move 1 to introduce a new circle $J$ with framing \pm 1 . The characteristic sublink of the enlarged link is $\{K, J\}$. Add a push-off of $J$ to $K$ (Move 2) and denote the result by $\bar{K}$. The single component $\bar{K}$ is now characteristic since $[\bar{K}]=[K]+[J]$. As

$$
\operatorname{fr} \bar{K}=[\bar{K}] \cdot[\bar{K}]=([K]+[J]) \cdot([K]+[J])=[K] \cdot[K] \pm 1,
$$

the reduction is complete.

Case 3. $L^{1}$ consists of a single component $K$.

It is well known that any knot can be trivialized by changing some of its crossings in a regular projection. The proof proceeds by induction on the number of such changes necessary to make $K$ trivial. Case 2 begins the induction.

Let $K^{1}$ be a knot obtained from $K$ by changing a crossing such that $K^{1}$ can be trivialized by changing one fewer crossing than can $K$. The induction will be completed by using the lemmas in $\$ 2$ to replace $L$ by a framed link in which $K^{1}$ is characteristic.

According to Lemma $2.1, K$ may be replaced in $L$ by a copy of $K^{1}$ along with a trivial circle $J$ which spans a disk $D$ intersecting $K^{1}$ in two points. Let $l$ be an arc in $D$ connecting the two points of intersection. It is clear that in the enlarged link $\left\{K^{1}, J\right\}$ is characteristic. Choose a 3-ball $B \subset S^{3}$ such that

(i) $\partial l \subset K^{1} \cap B^{3}=K^{1} \cap \partial B$,

(ii) $K^{1} \cap \partial B$ and $l \cap\left(S^{3}-B\right)$ are both connected.

See Diagram 3.2. 


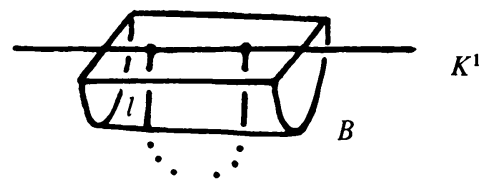

Diagram 3.2

Claim 1. It can be assumed that $l \subset B$.

Proof of Claim 1. The obstruction to realizing the claim by an isotopy is that $\overline{\left(K^{1} \cap\left(S^{3}-B\right)\right)}$ and $\overline{\left(l \cap\left(S^{3}-B\right)\right)}$ may be linked in $\overline{\left(S^{3}-B\right)}$. Such linking can be removed by changing crossings at which one strand is part of each arc. Recall that such a crossing corresponds to a crossing of two strands of $J$ (one on each side of $D$ ) with a strand of $K^{1}$. Apply Lemma 2.2 to change such a crossing at the expense of introducing a new component and a full twist of $D$ around $l$. Each such modification changes $L, K^{1}$, and $J$; however, we continue to use the same names. Because push-offs of the new components are added an odd number of times, the new components do not become part of the characteristic sublink. Thus, the modified sublink $\left(K^{1}, J\right)$ is characteristic in the modified $L$. The proof of Claim 1 is complete.

Claim 2. It can be assumed that $l \subset B$ is unknotted.

Proof of Claim 2. It is sufficient to change crossings of one strand of $l$ with another. Such a crossing represents two strands of $J$ crossing two other strands. It can be regarded as a pair of two over one crossings. Such crossings can, therefore, be changed by two applications of Lemma 2.2. As in the proof of Claim 1, $\left\{K^{1}, J\right\}$ remains characteristic. This completes the proof of Claim 2.

Since $l \subset B$ is unknotted, $l \cup\left(K^{1} \cap \partial B\right)$ bounds a 2-disk $E \subset B$. If $E \cap j=\varnothing, D$ could be isotoped along $E$ to a disk which does not intersect $K^{1}$. In this event, the arguments of Case 2 could be applied to remove $J$ from the characteristic sublink without disturbing $K^{1}$. This would complete the induction step.

The obstruction to finding a disk $E$ which misses $J$ is the number of half-twists of $D$ around $l$. See Diagram 3.3.

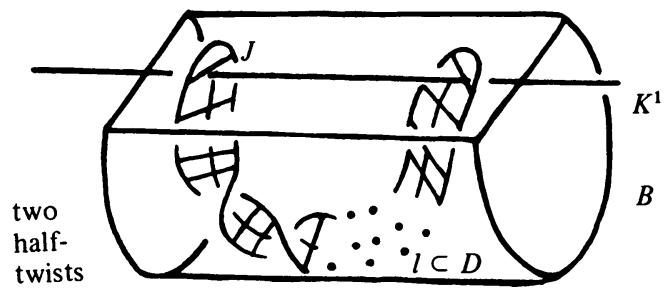

Diagram 3.3 
However, Lemma 2.3 shows how to remove a half-twist at the expense of introducing a new component. Since push-offs of the new component are added an odd number of times in Lemma 2.3, the new component does not become part of the characteristic sublink. This completes the proof of Case 3.

Case 4. $L^{1}$ consists of components $K_{1}, \ldots, K_{n}$.

Add a push-off of each $K_{2}, \ldots, K_{n}$ to $K_{1}$ (Move 2) and denote the result by $\bar{K}$. Then $[\bar{K}]=\left[K_{1}\right]+\cdots+\left[K_{n}\right]$ is characteristic. This reduces Case 4 to Case 3 and completes the proof of the theorem.

Example 3.2. The torus $T^{3}$ is $M_{L}$ for framed link $L$ shown in Diagram 3.4.

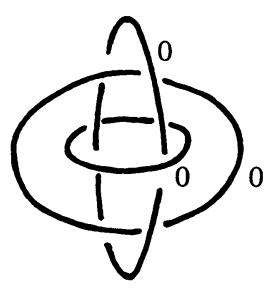

Diagram 3.4

By Remarks 1.8 and 1.11, $T^{3}$ has eight distinct almost framings and each sublink of $L$ is characteristic for one of these almost framings. Using the techniques of the proof of Theorem 3.1, it is easy to find framed 4-manifolds of index zero bounded by $T^{3}$ with seven of its almost framings and a framed 4-manifold of index 8 bounded by $T^{3}$ with the other almost framing. Since by a theorem of Rohlin [6], there exists no closed, almost framed 4-manifold of index 8, this reflects an important disparity between seven of the almost framings of $T^{3}$ and the other. In $\$ 6$ we show that the almost framings of a large class of closed, oriented 3-manifolds exhibit a similar phenomenon (Theorem 6.12).

THEOREM 3.3. There exists a closed, almost parallelizable 4-manifold with index $=16$ and $\beta_{2}=22$ which admits $a$ handlebody presentation with no handles of index 1 or 3 .

REMARK 3.4. The manifold obtained in Theorem 3.3 is homotopy equivalent to the Kummer surface, a complex surface well known to algebraic geometers. There is no known closed, almost parallelizable 4-manifold with index $=16$ and $\beta_{2}<22$. Recently, Harer, Kas and Kirby [3] have produced a handlebody presentation of this type for the Kummer surface. (Example 4.4 will give an alternate proof of Theorem 3.3 via Theorem 4.2.)

Proof of Theorem 3.3. A picture proof is given in Diagram 3.5. 

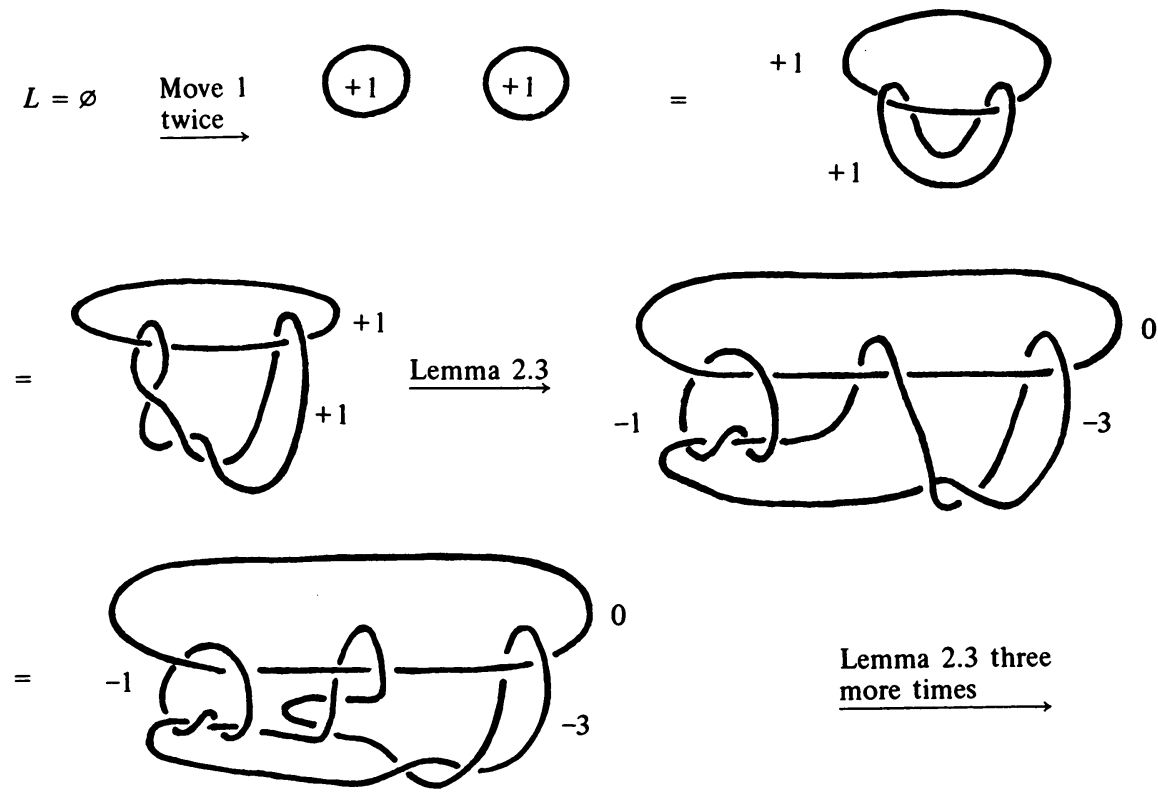

Lemma 2.3 three more times

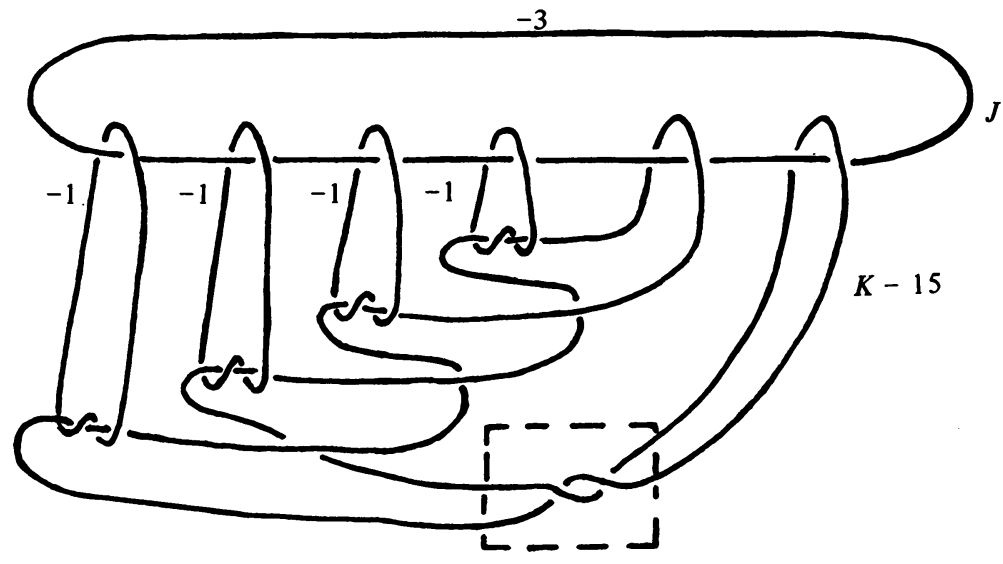

region in box $=$

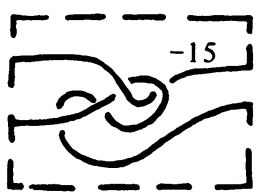

$\stackrel{\text { Lemma } 2.3}{\longrightarrow}$

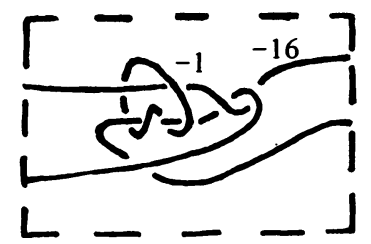

Diagram 3.5 
After the modification in the boxes above is made in the large picture, the characteristic sublink $\{J, K\}$ becomes an unlink. The manifold obtained by applying the arguments of Case 2 to $J$ and $K$ can be capped off with a ball to yield the desired handlebody.

4. More framed manifolds. Let $M$ be a closed, almost framed 3-manifold and suppose that $M=M_{L}$ for some framed link $L$ in which a single component $K$ is characteristic. The main result of this section (Theorem 4.2) is a procedure for finding a framed, 1-connected 4-manifold with comparatively low second betti number bounded by $M$. A formula is given which allows the computation of the index and second betti number of the framed 4-manifold without actually producing that manifold. The formula depends upon the number of components of $L$ and the framing, genus, and $\mu$-invariant of $K$. Most of the framed 4-manifolds obtained are 4-manifolds associated to framed links (Definition 1.3) and explicit link presentations can be found by carrying out the steps of the proof.

LEMMA 4.1. Suppose $K_{1}$ and $K_{2}$ are components of a framed link $L$ and that $K_{1}$ has framing zero and spans a disk intersecting $K_{2}$ in a single point. Then by adding push-offs of $K_{1}$ to the components of $L, K_{2}$ can be unknotted and unlinked from all components of $L$ except $K_{1}$.

Proof. The knotting and linking can be removed by changing crossings at which one or both of the strands is part of $K_{2}$. Slide $K_{1}$ along $K_{2}$ to a crossing which needs to be changed. Add a push-off of $K_{1}$ to the other strand at the crossing as shown in Diagram 4.1.
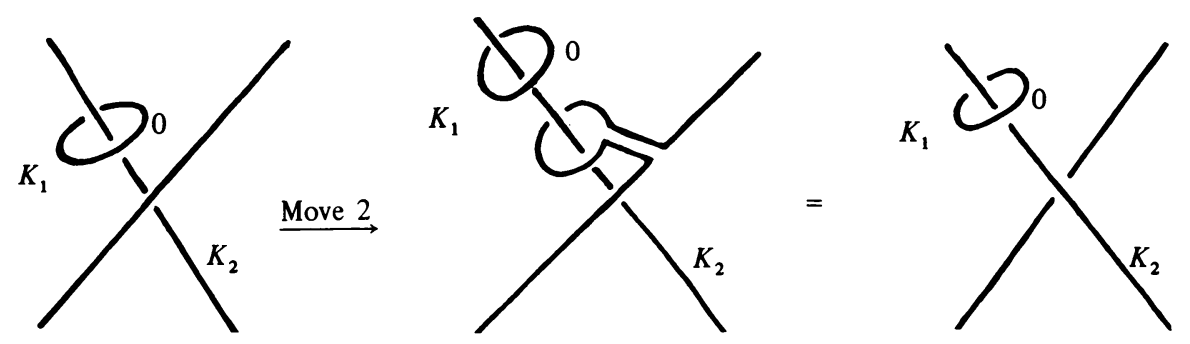

Diagram 4.1

While the crossing has been changed, $K_{1}$ still satisfies the original hypotheses. Induction on the number of crossings to be changed completes the proof.

We denote by $h(K)$ the genus of $K$. 
Theorem 4.2. Suppose $L$ is a framed link in $S^{3}$ in which a component $K$ is characteristic. Then $M_{L}$ (with the almost framing given by $K$ ) bounds a simply connected framed 4-manifold $W$ such that

(i) $\beta_{2}(W)=\beta_{2}\left(W_{L}\right)+|| \operatorname{fr}(K) \pm 8 \mu(K)|-1|+2 h(K)+4 \mu(K)-1$.

(ii) index $(W)=$ index $\left(W_{L}\right)-(\operatorname{fr}(K) \pm 8 \mu(K))$.

Moreover,

(iii) $W=W_{L^{1}}$ for some framed link $L^{1}$.

Addendum. If (iii) is omitted from the conclusion, then other 4 manifolds may be obtained such that $h(K)$ in (i) is replaced by $h\left(K^{1}\right)$ where $K^{1}$ is any knot cobordant to $K$.

Proof.

Case 1. $K$ is the trivial knot.

The manifold obtained from Case 2 of the proof of Theorem 3.1 is as required.

Case 2. $\mu(K)=0$.

The proof proceeds by induction on $h(K)$. The induction begins with Case 1 above. To complete the induction step it is sufficient to find a framed link $\hat{L}$ with characteristic knot $\hat{K}$ such that:

(a) $M_{\hat{L}}=M_{L}$,

(b) $\beta_{2}\left(W_{\hat{L}}\right)=\beta_{2}\left(W_{L}\right)+2 ; \operatorname{index}\left(W_{\hat{L}}\right)=\operatorname{index}\left(W_{L}\right)$,

(c) $\operatorname{fr}(\hat{K})=\operatorname{fr}(K) ; h(\hat{K})=h(K)-1$.

We adopt the notation of Definition 1.12. By Remark 1.13, $K$ has a minimal Seifert surface $F$ with canonical curves $a_{1}, b_{1}, \ldots, a_{h(K)}, b_{h(K)}$ such that $\alpha_{i}=0 \bmod 2$ for $1<i<h(K)$. We regard $F$ as a disk with bands with each $a_{i}$ and $b_{i}$ the core of a band. Enlarge $L$ by adding a pair of circles as shown in Diagram 4.2.

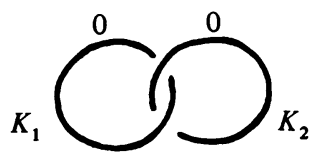

Diagram 4.2

This operation corresponds to replacing $W_{L}$ by $W_{L} \# S^{2} \times S^{2}$. The associated 3-manifold does not change and $K$ remains characteristic. Add a push-off of $K_{1}$ to each of the two strands of $K$ along $a_{1}$ (see Diagram 4.3).

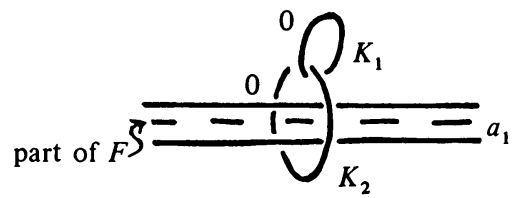

Diagram 4.3 
Since $F \cap K_{2}=\varnothing, \operatorname{lk}\left(K, K_{2}\right)=0$.

Now $K_{2}$ links $a_{1}$ in the manner required by Lemma 4.1. By adding push-offs of $K_{2}$ to $K$ in two places, one can, in effect, add a push-off of $K_{2}$ to an $a_{i}$ or $b_{j}$. Thus, Lemma 4.1 can be applied to unknot $a_{1}$ and unlink it from the other $a_{i}$ and the $b_{j}$ without changing $M_{L}$. Since Move 2 is applied an even number of times and $\operatorname{lk}\left(K_{2}, K\right)=0$, the curve taking the place of $K$ remains characteristic and its framing is unchanged. Diagram 4.4 shows how to add a push-off of $K_{2}$ to $K$ to change $\alpha_{1}$ by \pm 1 . Since $\alpha_{1}=0 \bmod 2$, this can be done an even number of times to make $\alpha_{1}=0$.

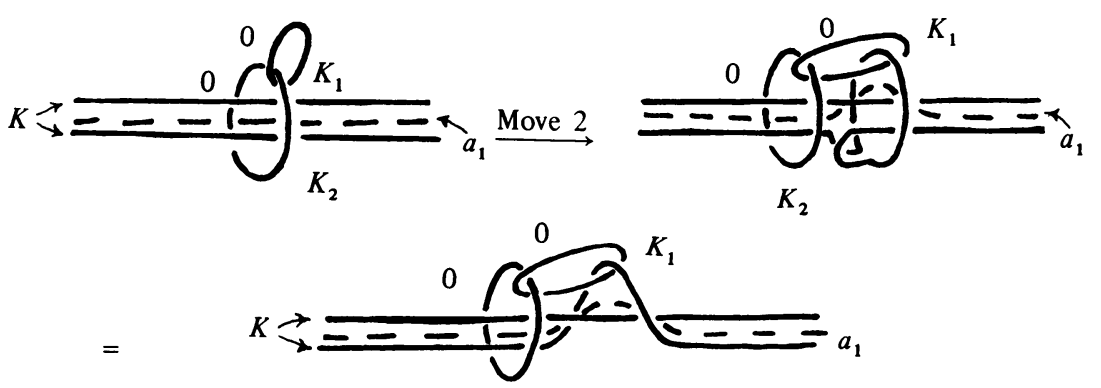

Diagram 4.4

Let $\hat{L}$ be the framed link obtained from the enlargement of $L$ by applying Move 2 to unknot $a_{1}$, unlink it from the $a_{i}$ and $b_{j}$ and make $\alpha_{1}=0$. Let $\hat{K}$ be the curve in $\hat{L}$ which results from applying these moves to $K$. All of the properties of $(\hat{L}, \hat{K})$ needed to complete the induction step have been verified except that $h(\hat{K})=h(K)-1$. Diagram 4.5 shows why this is true and completes Case 2.
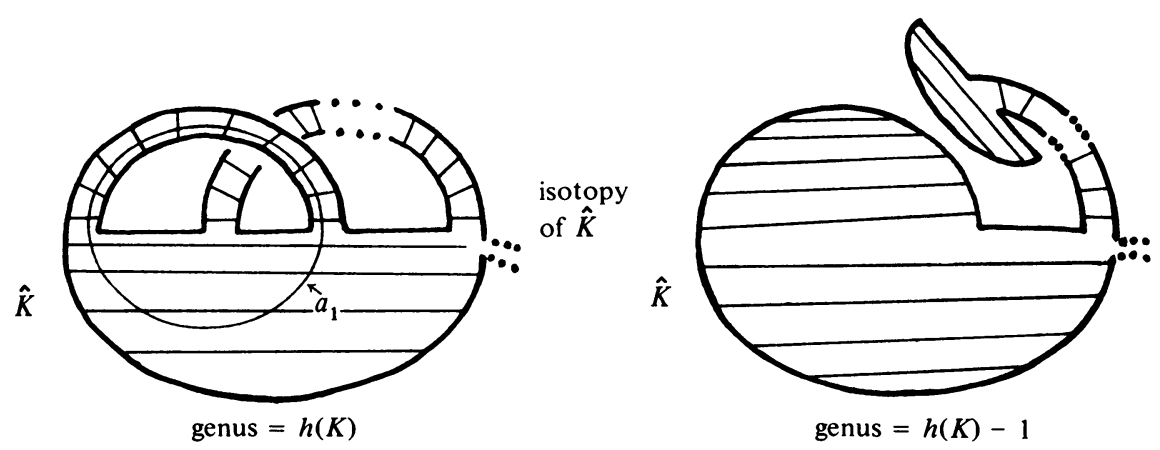

Diagram 4.5

Case 3. $\mu(K)=1$.

To reduce Case 3 to Case 2 it is sufficient to find a framed link $\hat{L}$ with characteristic knot $\hat{K}$ such that: 
(a) $W_{\hat{L}}=W_{L}$,

(b) $\beta_{2}\left(W_{\hat{L}}\right)=\beta_{2}\left(W_{L}\right)+2 ;$ index $\left(W_{\hat{L}}\right)=\operatorname{index}\left(W_{L}\right)$,

(c) $\operatorname{fr}(\hat{K})=\operatorname{fr}(K) \pm 8 ; h(\hat{K})=h(K)+1 ; \mu(\hat{K})=0$.

It is shown in Diagram 4.6 that the right-handed trefoil knot $T$ is characteristic with framing 8 in a link $\bar{L}$ with two components where $M_{\bar{L}}=S^{3}$. The left-handle trefoil knot is characteristic in an analogous link with framing -8 . To satisfy the conditions above let $\hat{L}=L \cup \bar{L}, \hat{K}=K \# T$. This completes the proof of the theorem.

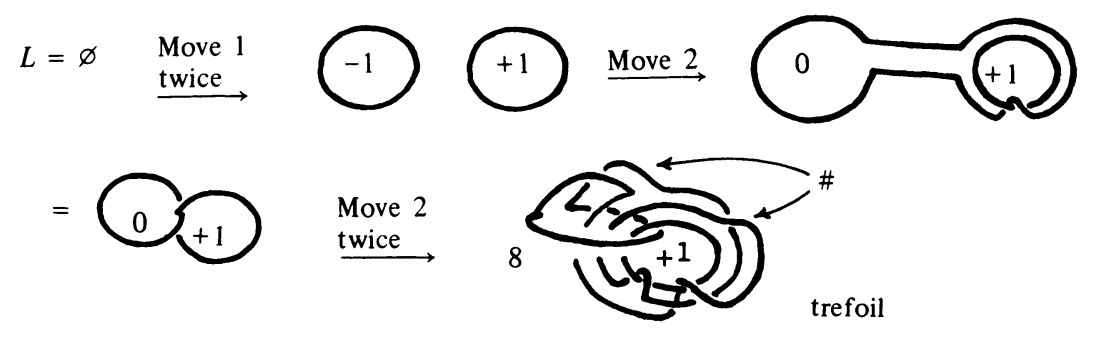

Diagram 4.6

Proof OF THE ADDENDUM. It is sufficient to consider the case $\mu(K)=0$, for the arguments of Case 3 will again apply. Suppose $K$ is cobordant to $K^{1}$. Embed the cobordism in a collar of $S^{3}=\partial B^{4} \subset W_{L}$. The argument proceeds as in Case 2 except that new pairs of handles (as represented by the link in Diagram 4.2) are added at the level in which $K^{1}$ occurs and are used to decrease the genus of $K^{1}$. Since $K_{1}$ and $K_{2}$ (notation from Diagram 4.2) and their push-offs bound disjoint smooth disks in $W_{L} \# S^{2} \times S^{2}$ which are disjoint from the cobordism, $K$ is cobordant to the connected sum of $K^{1}$ with any collection of push-offs of $K_{1}$ and $K_{2}$. In the end, the characteristic class can be represented by a smooth 2-sphere. Apply Case 1 so that the normal bundle of the 2 -sphere has Euler number \pm 1 . The boundary of the normal disk bundle is then $S^{3}$ and replacing the disk bundle by a 4-ball gives the desired manifold.

REMARK 4.3. The $\mu(K)=0$ case of the addendum is implicit in the proof of a result of C. McA. Gordon, [2, Theorem 1.]

ExAmple 4.4. As shown in Diagram 4.7, the (2.7) torus knot with framing 16 is characteristic in a framed link $L$ with two components such that $M_{L}=S^{3}$. An application of Theorem 4.2 yields a framed link $L^{1}$ such that $W_{L^{1}}$ is parallelizable, index $W_{L^{1}}=16, \beta_{2}\left(W_{L^{1}}\right)=22$ and $M_{L}=S^{3}$. Then $W_{L^{1}} \cup S_{S^{3}} B^{4}$ is a closed almost parallelizable 4-manifold with index $=16$ and $\beta_{2}=22$ which admits a handle decomposition with no handles of index 1 or 3 . 


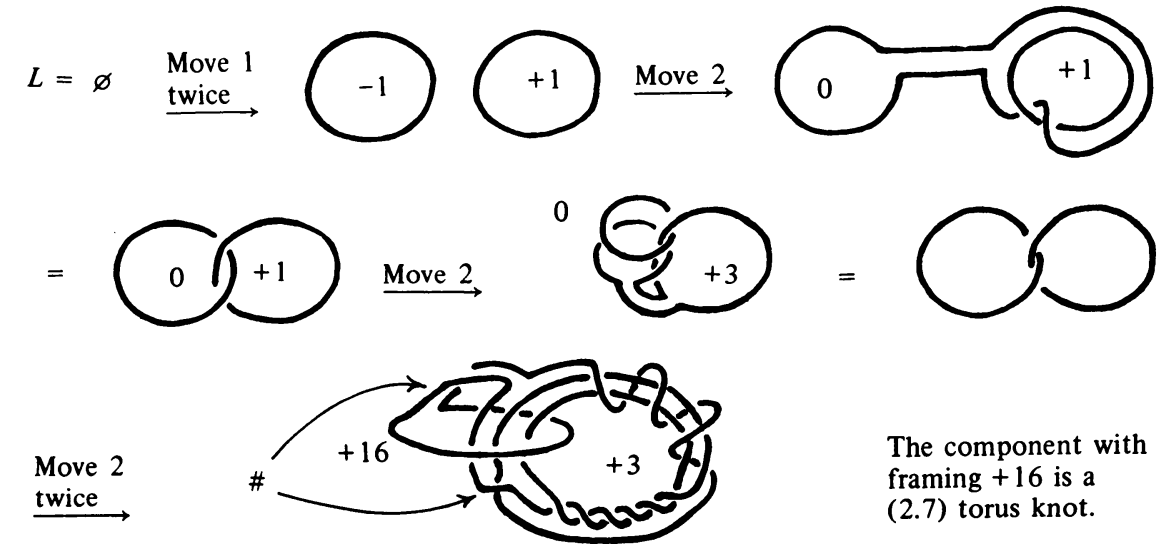

Diagram 4.7

5. A new description of $\mu(K)$. In order to apply Theorem 4.2 to find the index and second betti number of a framed 4-manifold with given boundary, it is necessary to evaluate the $\mu$-invariant of a characteristic knot. In this section, a new definition of the invariant $\mu(K)$ is given. It is shown that every knot in $S^{2}$ spans a certain type of immersed 2-disk from which the $\mu$-invariant of the knot is easily determined. The first part of the section is devoted to singling out the type of immersion we wish to study.

Suppose $D^{2} \rightarrow S^{3}$ is an immersion whose self-intersections are transverse and include no triple points or closed components. Then each component of self-intersection is either a clasp or a ribbon (see Diagram 5.1).

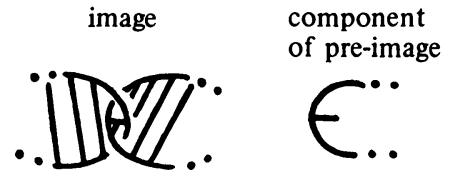

clasp
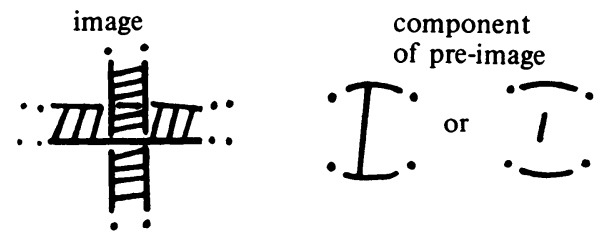

ribbon

Diagram 5.1

Next, suppose that $D^{2} \rightarrow S^{3}$ is an immersion as above except that:

(i) there may be triple points,

(ii) no intersection of two sheets is a clasp.

Suppose $p$ is a triple point and $l_{1}, l_{2}, l_{3}$ are the double lines intersecting at $p$. The pre-image of each $l_{i}$ consists of two segments, one contained in int $D^{2}$, the other properly embedded in $D^{2}$. We say that $p$ is a triple point of Type I if 
some component of the pre-image of $l_{1} \cup l_{2} \cup l_{2}$ is contained in int $D^{2}$. We say that $p$ is a triple point of Type II if each component of the pre-image of $l_{1} \cup l_{2} \cup l_{2}$ intersects $\partial D^{2}$. (See Diagram 5.2.)

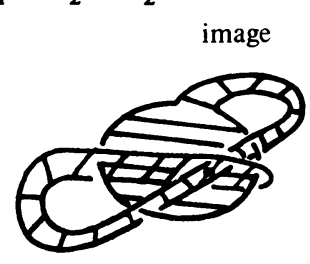

Triple point of Type I

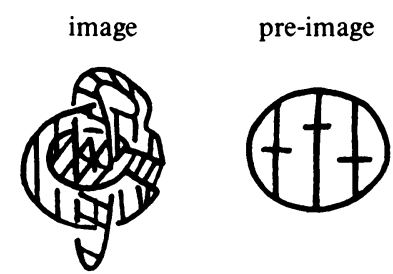

Triple point of Type II

Diagram 5.2

The triple points of Type I can be removed by a regular homotopy which restricts to an isotopy of the boundary and introduces no clasp intersections. This is not the case with a triple point of the second type.

Definition 5.1. A singular ribbon is a 2-disk immersed in $S^{3}$ whose self-intersections are ribbon intersections and triple intersections of Type II.

THEOREM 5.2. Every knot $K \subset S^{3}$ bounds a singular ribbon.

Proof. Suppose $K$ is a connected sum (along paths in $S^{3}$ ) of knots which span immersed disks whose self- and mutual intersections are ribbon intersections and triple intersections of Type II. By general position, the connecting paths can be chosen to miss the intersections of the disks. Then the (boundary) connected sum of the disks along the same paths is a singular ribbon with boundary $K$. (Each intersection of a connecting path with a disk creates a new ribbon intersection, but no clasps are introduced.) We will represent $K$ as such a connected sum.

It is well known that any knot can be trivialized by changing some of its crossings in a regular projection. The proof proceeds by induction on the number of crossings which need to be changed to make $K$ trivial.

The process of changing a crossing of a knot can be realized by taking the connected sum of the knot with a trivial circle linking the knot zero times algebraically and twice geometrically, as in Diagram 5.3.
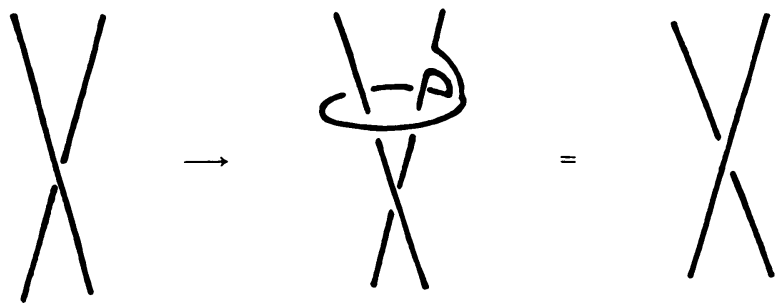

Diagram 5.3 
Thus, $K$ is the connected sum of a knot $K^{1}$, which, by the induction hypothesis, bounds a singular ribbon and a circle $J$ which spans a disk $D$ intersecting $K^{1}$ in two points (with opposite signs). Let $l_{1}$ be an arc in $D$ joining the two points of intersection and $l_{2}$ an arc in $K^{1}$ bounded by these two points.

First consider the case where $l_{1}$ is isotopic to $l_{2}$ by an isotopy of $S^{3}$ which fixes $K^{1}$. In this case we can assume that $D$ lies in a small regular neighborhood $B$ of $l_{2}$ and that inside $B$ the picture is that given in Diagram 5.4.

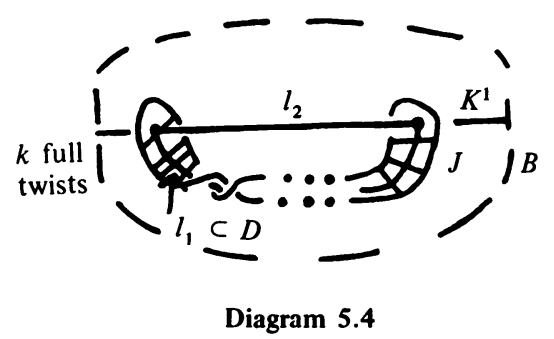

Since $\operatorname{lk}\left(J, K^{1}\right)=0$, there are an even number, say $2 k$, half-twists. Removing these twists amounts to changing $k$ crossings, so $J$ can be viewed as a connected sum of the components of the link in Diagram 5.5.

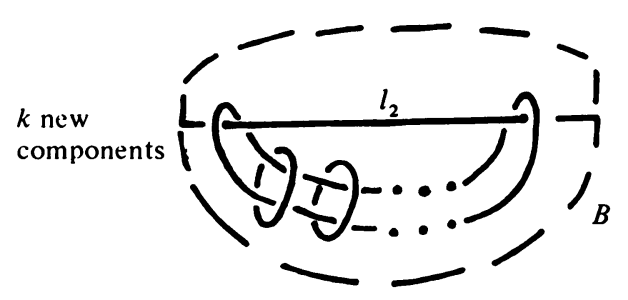

Diagram 5.5

It is easy to split $K^{1}$ as the connected sum of a trivial knot in $B$ and a knot $\bar{K}^{1}$ outside $B$, as in Diagram 5.6.

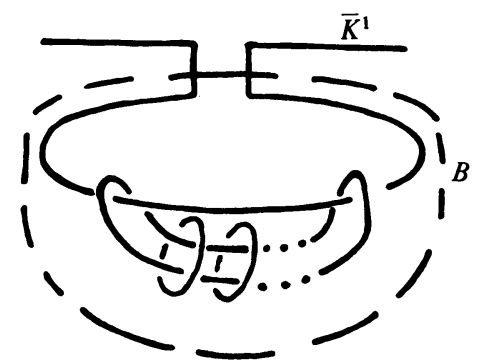

Diagram 5.6 
Since $\bar{K}^{1}$ is isotopic to $K^{1}$, it bounds a singular ribbon which can be chosen to lie outside $B$. The components of the link inside $B$ bound disks whose mutual intersections are all triple intersections of Type II (see Diagram 5.7).
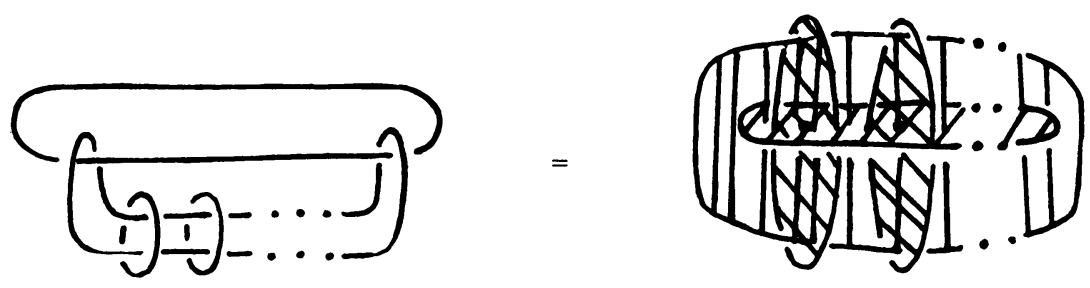

Diagram 5.7

This completes the proof in the special case.

For the proof in the general case, we again show that $K$ bounds a singular ribbon which is the connected sum of a singular ribbon whose boundary is isotopic to $K^{1}$ and disks of the type pictured in Diagram 5.7. To realize the hypothesis of the special case, it suffices to be able to change crossings of two types:

(i) one strand is part of $K^{1}$ and the other is part of $l_{1}$;

(ii) each strand is part of $l_{1}$.

To change a crossing of the first type, split $K^{1}$ as the connected sum of a knot with the crossing changed and a small circle around $l_{1}$ (such as the ones in Diagram 5.5); see Diagram 5.8.

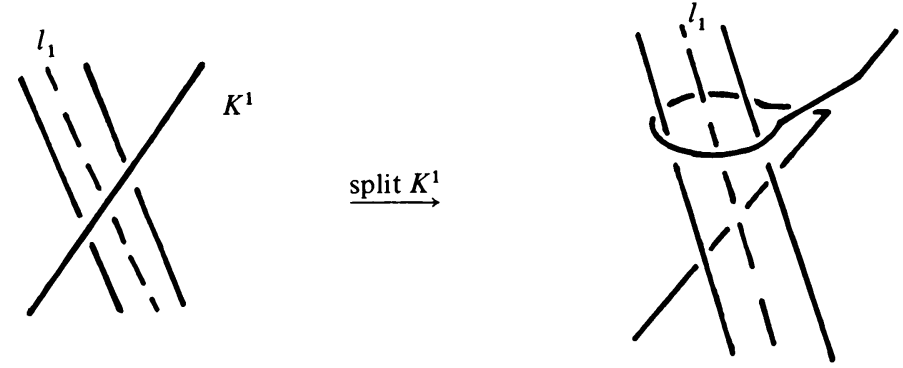

Diagram 5.8

A crossing of the second type represents a pair of strands of $J$ crossing a strand of $l_{1}$. Regard $J$ as the connected sum of its analogue with the crossing changed with each of two circles around $l_{1}$. After the appropriate crossings are changed, the hypothesis of the special case is fulfilled and the proof may be completed in the same way. (The additional circles around $l_{1}$ pose no problem.)

THEOREM 5.3. Let $E$ be a singular ribbon with $k$ triple points. Then $\mu(\partial E)=$ $[k] \in Z_{2}$. 


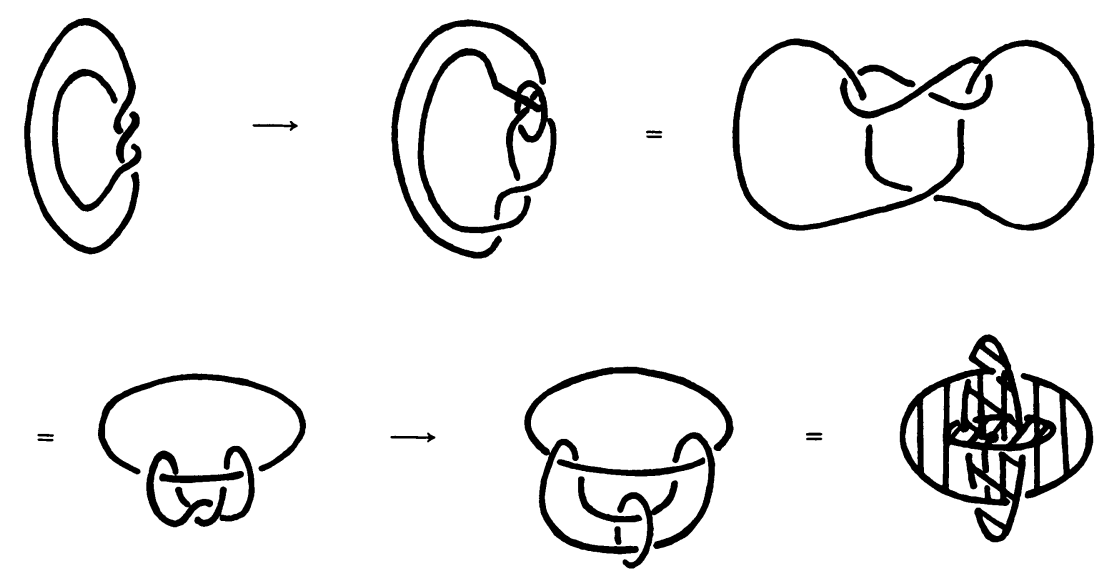

Diagram 5.9
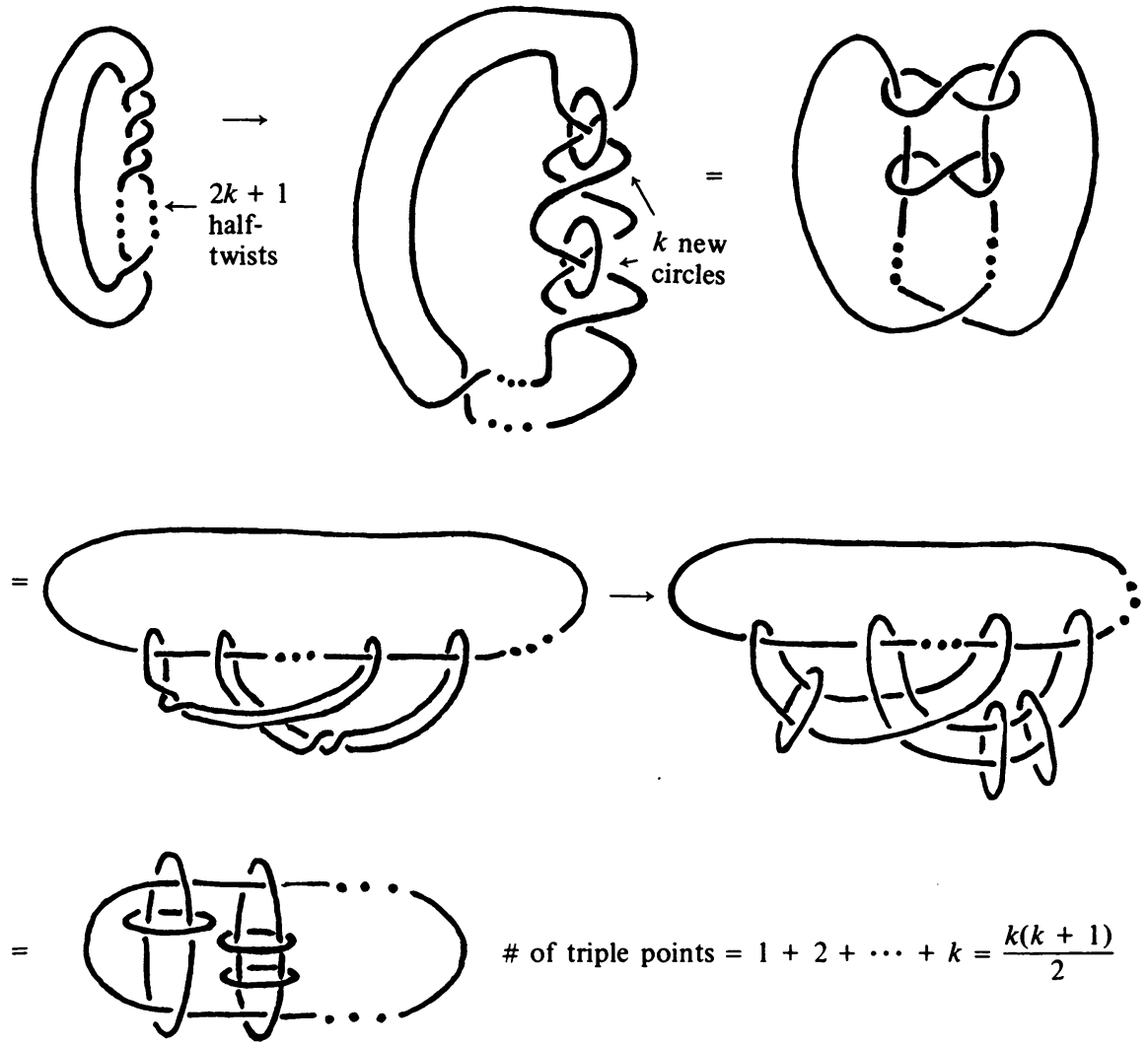

Diagram 5.10 
Proof. According to Robertello [8], if two knots are cobordant by disks with holes to the same proper link (the linking number of any pair of components is even), they have the same $\mu$-invariant. By slicing away the ribbon intersections of $E$ in the usual way, it is easy to show that $\partial E$ is cobordant by a disk with holes to $k$ copies of the Borromean rings. A connected sum of $k$ knots where each is a trefoil or a figure 8 is cobordant to the same oriented link. The $\mu$-invariant of this knot is $[k] \in Z_{2}$.

The remainder of the section is devoted to examples which show how to find a singular ribbon with given boundary. An arrow $(\rightarrow)$ will denote decomposition as a connected sum.

Example 5.4. The trefoil knot. See Diagram 5.9.

Example 5.5. The $(2,2 k+1)$-torus knot. See Diagram 5.10.

EXAMPLE 5.6. The rational knot of type $(p, q)$. See Diagram 5.11.

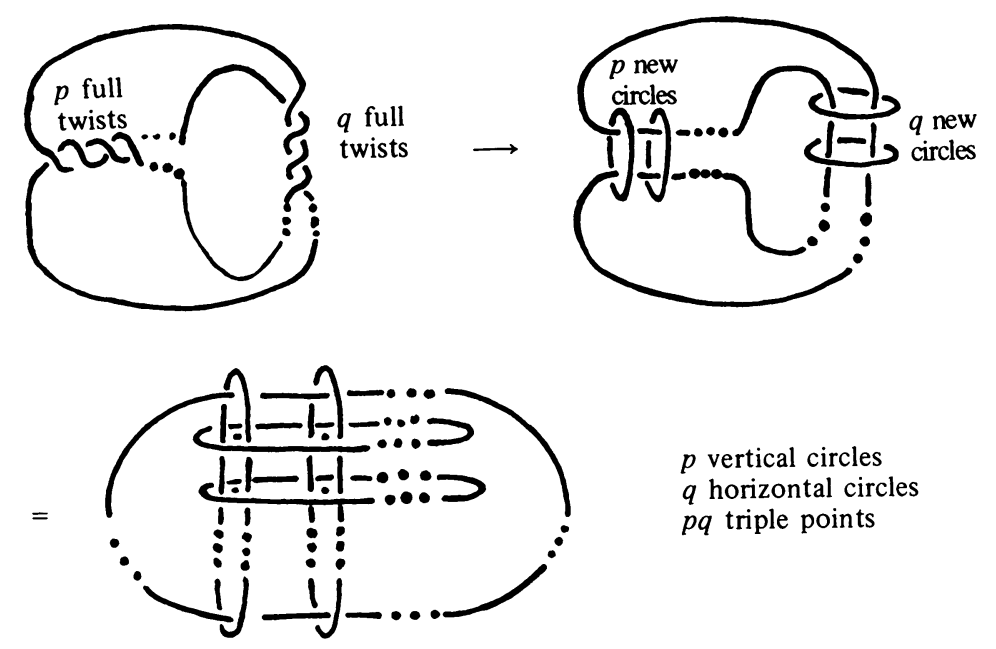

Diagram 5.11

6. Triple points and framed manifolds. In Example 3.2, it was noted that the 3-dimensional torus $T^{3}$ bounds a framed manifold of index 0 with seven of its eight almost framings and a manifold of index 8 with the other almost framing. The main result of this section is that there is a large class of closed, oriented 3-manifolds with a similar property:

Theorem 6.12. Suppose $M$ is a closed, connected oriented 3-manifold which bounds a framed manifold of index $k$. Suppose further that there exist elements $x, y, x \in H^{1}(M)$ such that $x \cup y \cup z$ is an odd multiple of the generator of $H^{3}(M)$. Then $M^{3}$ bounds a framed manifold of index $k+8$. 
Together with the Kervaire-Milnor Theorem [5], Theorem 6.12 can be used to detect the existence of classes in many bounded 4-manifolds which cannot be represented by smoothly embedded 2 -spheres. In order to prove Theorem 6.12 , a theory of singular ribbons is developed for links in which the linking number of any pair of components is zero. This leads to the definition of invariants of such a link which are related, on the one hand, to the $\mu$-invariants of the various connected sums of the components, and on the other hand, to the cup product structure of the 3-manifold associated to the link where each component has framing zero (Definition 1.3). The section begins with an analogue of Theorem 5.2 for links.

THEOREM 6.1. Suppose $L \subset S^{3}$ is a link with components $K_{1}, \ldots, K_{n}$ which satisfy $\operatorname{lk}\left(K_{i}, K_{j}\right)=0$ for all $1 \leqslant i, j \leqslant n$. Then $K_{1}, \ldots, K_{n}$ bound singular ribbons such that each component of the mutual intersection is a ribbon intersection or a triple intersection of Type II (Diagram 5.2).

Proof. As in the proof of Theorem 5.1, it is permissible to decompose the $K_{i}$ as connected sums of components of a larger link and to prove the theorem for the larger link. It is not difficult to see that the $K_{i}$ are connected sums of trivial knots which span disks such that, aside from ribbon intersections, any two disks are either disjoint or intersect in two clasps. These pairs of clasps are removed by introducing triple intersections just as in the proof of Theorem 5.1. The details are left to the reader.

Suppose $K_{1}, \ldots, K_{n}$ are such that $\operatorname{lk}\left(K_{i}, K_{j}\right)=0$ for all $1<i, j<n$ and $D_{1}, \ldots, D_{n}$ are singular ribbons satisfying the conclusion of Theorem 6.1.

Definition 6.2. The element $(i, j, k)$ of $Z_{2}$ is the mod 2 reduction of the number of points in $D_{i} \cap D_{j} \cap D_{k}$.

LEMMA 6.3. The following equation is valid (in $\left.Z_{2}\right)$ :

$$
\begin{aligned}
(i, j, k)= & \mu\left(K_{i}\right)+\mu\left(K_{j}\right)+\mu\left(K_{k}\right)+\mu\left(K_{i} \# K_{j}\right)+\mu\left(K_{i} \# K_{k}\right)+\cdots \\
& +\mu\left(K_{j} \# K_{k}\right)+\mu\left(K_{i} \# K_{j} \# K_{k}\right) .
\end{aligned}
$$

Proof. According to Theorem 5.3,

$$
\begin{gathered}
\mu\left(K_{p}\right)=(p, p, p) ; \\
\mu\left(K_{p} \# K_{q}\right)=(p, p, p)+(p, p, q)+(p, q, q)+(q, q, q) ; \\
\mu\left(K_{p} \# K_{q} \# K_{r}\right)=(p, p, p)+(q, q, q)+(r, r, r)+(p, p, q)+(p, q, q) \\
+(p, p, r)+(p, r, r)+(q, q, r)+(q, r, r)+(p, q, r) .
\end{gathered}
$$

The lemma follows easily.

Suppose that $L \subset S^{3}$ is an oriented link with components $K_{1}, \ldots, K_{n}$ satisfying $\operatorname{lk}\left(K_{i}, K_{j}\right)=0$ for all $1 \leqslant i, j \leqslant n$. Let $F_{1}, \ldots, F_{n}$ be Seifert surfaces for $K_{1}, \ldots, K_{n}$ satisfying $F_{i} \cap L=K_{i}$. Let $v_{1}, \ldots, v_{n}$ be normal 
vector fields to $F_{1}, \ldots, F_{n}$ satisfying:

(i) at each $p \in F_{i} \cap F_{j}$, $\left(v_{i}\right)_{p}$ is tangent to $F_{j}$;

(ii) at each $p \in K_{i}$, the ordered triple consisting of a positively oriented tangent vector to $K$ at $p$, an inward pointing normal (towards $F_{i}$ ) at $p$, and $\left(v_{i}\right)_{p}$ is a positively oriented frame in $S^{3}$.

Assume $i, j$ and $k$ are distinct.

A sign $( \pm 1)$ is associated to each point $p \in F_{i} \cap F_{j} \cap F_{k}$ by comparing the frame $\left(\left(v_{i}\right)_{p},\left(v_{j}\right)_{p},\left(v_{k}\right)_{p}\right)$ with the orientation on $S^{3}$.

Definition 6.4. Assume the notation above. The integer $T(i, j, k)$ is equal to the sum of the signs of the points in $F_{i} \cap F_{j} \cap F_{k}$.

Proposition 6.5. $T(i, j, k)$ is well-defined.

Proof. By symmetry, it suffices to show that $T(i, j, k)$ does not depend on the choice of $F_{i}$. Note that $T(i, j, k)=\operatorname{lk}\left(K_{i}, F_{j} \cap F_{k}\right)$. Thus, $T(i, j, k)$ is indeed independent of the choice of $F_{i}$.

Proposition 6.6. Suppose $L$ is as above and $i, j$ and $k$ are distinct. Then the $\bmod 2$ reduction of $T(i, j, k)$ is equal to $(i, j, k)$.

Proof. It was noted in the proof of Proposition 6.5 that $T(i, j, k)$ may be defined as a linking number. Thus, to compare $T(i, j, k)$ it is sufficient to find immersed surfaces $F_{i}, F_{j}$ and $F_{k}$ which satisfy $F_{p} \cap L=K_{p}$. Suppose $D_{i}, D_{j}$ and $D_{k}$ are singular ribbons with boundaries $K_{i}, K_{j}$, and $K_{k}$ whose mutual intersections are all ribbon intersections and triple intersections of Type II. We show how to remove pairs of points of $D_{p} \cap K_{q}$ at the expense of replacing $D_{p}$ by an immersed surface of larger genus. Let $l$ be a line $D_{p} \cap D_{q}$ such that $\partial l \subset K_{q}$. Replace a neighborhood of $\partial l$ in $D_{p}$ by a tube running along $K_{q}$. Since there are no triple points of type I (Diagram 5.2), this process can be iterated without introducing any additional triple points. Thus, $D_{i}, D_{j}$ and $D_{k}$ can be replaced by immersed surfaces $F_{i}, F_{j}$ and $F_{k}$ such that $F_{p} \cap L=K_{p}$ and $\#\left(D_{i} \cap D_{j} \cap D_{k}\right)=\#\left(F_{i} \cap F_{j} \cap F_{k}\right)$. The result is now clear.

Definition. A link $L \subset S^{3}$ is slice in the strong sense if the components of $L$ bound disjoint smooth 2-disks in $B^{4}$.

THEOREM 6.7. Suppose $L$ is a link in $S^{3}$ which is slice in the strong sense. Then $T$ is identically zero on $L$.

Proof. Let $K_{1}, \ldots, K_{n}$ be the components of $L$ and $D_{1}, \ldots, D_{n}$ the disjoint smooth disks they bound in $B^{4}$. Choose Seifert surfaces $F_{1}, \ldots, F_{n}$ for the $K_{i}$ which satisfy $F_{i} \cap L=K_{i}$. Let $N_{i}$ be a tubular neighborhood of $D_{i}$. Since $B^{4}-D_{i}$ is a cohomology circle for each $i$ and $S^{1}=K(Z, 1)$, there exist embedded, oriented 3-manifolds $M_{1}, \ldots, M_{n}$ in $B^{4}$ such that $\partial M_{i}=F_{i} \cup D_{i}$. 
Suppose $M_{i} \cap D_{j} \neq \varnothing$ for $i \neq j$. After making $M_{i}$ transverse to $\partial N_{j}, M_{i} \cap$ $\partial N_{j}$ will consist of a finite collection of closed 2-manifolds. Since any closed 2-manifold in $N \approx S^{1} \times D^{2}$ bounds an oriented 2-manifold, $M_{i}$ can be replaced by a 3-manifold which intersects $\partial N_{j}$ in one less component. (By dealing with an "innermost component" first, one can assure that the new $\boldsymbol{M}_{j}$ will be embedded.) Assume, then, that $M_{i} \cap D_{j}=\varnothing$ if $i \neq j$.

We can assume that the $M_{i}$ meet transversely. Each component of $M_{i} \cap M_{j}$ $\cap M_{k}$ which meets $\partial B^{4}$ must be a line segment and its boundary must consist of two points of $F_{i} \cap F_{j} \cap F_{k}$. Since $M_{i}, M_{j}$ and $M_{k}$ are oriented, the signs of these two triple points differ and, together, they contribute nothing to $T(i, j, k)$. Since $F_{i} \cap F_{j} \cap F_{k}=\partial\left(M_{i} \cap M_{j} \cap M_{k}\right)$, the proof is complete.

Lemma 6.3 and Proposition 6.6 show how $T(i, j, k)$ relates to the $\mu$-invariant of connected sums of components of a link in which all linking numbers are zero. We next exhibit its relationship to another known invariant. For any closed oriented 3-manifold $M$ there is an alternating form defined on $H_{2}(M)$ by representing the classes by immersed submanifolds intersecting transversely and taking the signed sum of the triple points. This form is dual to the triple cup product map: $H^{1}(M) \times H^{1}(M) \times H^{1}(M) \rightarrow$ $H^{3}(M)$ followed by evaluation on the fundamental class of $M$ and has been studied by Sullivan [10].

Suppose $W$ is a compact oriented 4-manifold with boundary $M$. Consider the image of the homomorphism $i_{*}: H_{2}(M) \rightarrow H_{2}(W)$. Since $M$ is collared in $W$, if $x \in \operatorname{im}\left(i_{*}\right)$, then $x \cdot y=0$ for each $y \in H_{2}(W)$.

Conversely, suppose $L$ is a framed link and $K$ is a component of $L$ such that $[K] \cdot x=0$ for all $x \in H_{2}\left(W_{L}\right)$. Then $[K]$ is the image under $i_{*}$ of a class $(K) \in H_{2}\left(M_{L}\right)$ represented in the following way: Span $K$ by a surface $F$ which misses the other components of $L$ and push $F$ off $K$ so that its boundary is a push-off of $K$ and $F \subset M_{L}$. Since $[K] \cdot[K]=0$, a push-off of $K$ bounds a disk over the handle in $M_{L}$.

Definition 6.8. Suppose $L$ is a framed link and $K$ is a component of $L$ such that $[K] \cdot x=0$ for all $x \in H_{2}\left(W_{L}\right)$. The class $(K) \in H_{2}\left(M_{L}\right)$ associated to $K$ is the class of the union of the surface $F$ and the disk described above.

THEOREM 6.9. Suppose $L$ is a framed link in which the components $K_{i}, K_{j}$, and $K_{k}$ each satisfy $\left[K_{p}\right] \cdot x=0$ for all $x \in H_{2}\left(W_{L}\right)$. Then $T(i, j, k)=\left(\left(K_{i}^{*}\right) \cup\right.$ $\left(K_{j}^{*}\right) \cup\left(K_{k}^{*}\right)$ ) evaluated on the fundamental class of $M$ (where $x^{*}$ is the Poincaré dual of $x$ ).

Proof. Represent $\left(K_{p}\right)$ as the union of a surface $F_{p}$ and disk over the handle above $K_{p}$ as in the discussion above for $p=i, j$ and $k$. Since the handles are disjoint, all of the triple points are points of $F_{i} \cap F_{j} \cap F_{k}$. These surfaces are suitable for computing $T(i, j, k)$. Thus, the result is clear. 
Suppose $L$ is a framed link in $S^{3}$ and consider the homology exact sequence of the pair $\left(W_{L}, M_{L}\right)$ :

$$
\ldots \rightarrow 0=H_{3}\left(W_{L}, M_{L}\right) \rightarrow H_{2}\left(M_{L}\right) \stackrel{i}{\rightarrow} H_{2}\left(W_{L}\right) \stackrel{j}{\rightarrow} H_{2}\left(W_{L}, M_{L}\right) \rightarrow \ldots
$$

The first group vanishes since $W_{L}$ is simply connected. The last two groups are free abelian. Note that $\operatorname{im}\left(i_{*}\right)$ and $H_{2}\left(W_{L}\right) / \operatorname{im}\left(i_{*}\right) \approx H_{2}\left(W_{L}\right) / \operatorname{ker}\left(j_{*}\right) \approx$ $\operatorname{im}\left(j_{*}\right)$ are subgroups of free abelian groups and are therefore both free abelian.

Lemma 6.10. Suppose $F$ is a free abelian group with a distinguished basis and $G$ is a subgroup of $F$ with free abelian quotient. Consider two types of modifications of the distinguished basis:

(i) replace an element $x$ by $-x$,

(ii) replace one element by its sum with a multiple of another element.

The basis can be modified in a combination of the two ways above to arrive at a new basis, a subset of which forms a basis for $G$.

Proof. Lemma 6.10 follows directly from Lemma 1 of $[4$, p. 357].

We now proceed to the main results of the section.

Proposition 6.11. Suppose $L$ is a framed link in $S^{3}$ and that there are elements $x, y$, and $z$ of $H^{1}\left(M_{L}\right)$ such that $x \cup y \cup z$ is an odd multiple of the generator of $H^{3}\left(M_{L}\right)$. Then there is a primitive class $\alpha \in H_{2}\left(W_{L}\right)$ with $\alpha \cdot \beta=$ 0 for all $\beta \in H^{2}\left(W_{L}\right)$ represented by an embedded 2-sphere which is smooth except at a single point where the link is a knot of $\mu$-invariant 1.

Proof. Again consider im $\left(i_{*}\right)$ where $i_{*}: H_{2}\left(M_{L}\right) \rightarrow H_{2}\left(W_{L}\right)$ is induced by the inclusion. By the remarks preceding Lemma 6.10, $H_{2}\left(W_{L}\right), \operatorname{im}\left(i_{*}\right)$ and $H_{2}\left(W_{L}\right) / \operatorname{im}\left(L_{*}\right)$ are all free abelian. If the components of $L$ are $K_{1}, \ldots, K_{n}$, the classes $\left[K_{i}\right]$ give a distinguished basis for $H_{2}\left(W_{L}\right)$. Note that the two operations allowed in Lemma 6.10 when applied to the $\left[K_{i}\right]$ correspond to changing the orientation on a component of $L$ and Move 2 (see $\$ 1$ ), respectively. They can, thus, be carried out geometrically by modifying $L$ without changing $M_{L}$. After applying Lemma 6.10 and carrying each step out geometrically we can assume that for some $k<n,\left[K_{1}\right], \ldots,\left[K_{k}\right]$ form a basis for $\operatorname{im}\left(i_{*}\right)$ and that, since $i_{*}$ is injective, $\left(K_{1}\right), \ldots,\left(K_{k}\right)$ form a basis for $H_{2}\left(M_{L}\right)$. If there exist classes $x, y, z$ as in the hypothesis, Theorem 6.9 and the remarks preceding Definition 6.8 show that there exist integers $1<p, q, r<$ $k$ such that $T(p, q, r)$ is odd. Thus, by Proposition 6.6, $(p, q, r) \neq 0$. By Lemma 6.3 some connected sum of $K_{p}, K_{q}$, and $K_{r}$ has nonzero $\mu$-invariant. The class in $H_{2}\left(W_{L}\right)$ associated to this connected sum is the desired class. 
THEOREM 6.12. Suppose $M$ is a closed, connected, oriented 3-manifold which bounds a framed manifold of index $k$. Suppose further that there exist elements $x, y, z \in H^{1}(M)$ such that $x \cup y \cup z$ is an odd multiple of the generator of $H^{3}(M)$. Then $M^{3}$ bounds a framed manifold of index $k+8$.

Proof. Let $W^{4}$ be the framed manifold. After a sequence of surgeries on $W^{4}$ to turn the handles of index 1 and 3 to handles of index $2, W^{4}=W_{L}$ for some framed link $L$. According to Remark 1.11, the class $\alpha$ given by Proposition 6.11 is characteristic for some almost framing on $M_{L}$. An application of Theorem 4.2 yields a framed manifold with boundary $M$ and index = $k+8$.

TheOREM 6.13. Suppose $W$ is a compact, parallelizable 4-manifold with connected boundary and that there exist classes $x, y, z \in H^{1}(\partial W)$ such that $x \cup y \cup z$ is an odd multiple of the generator of $H^{3}(\partial W)$. Then there is a primitive class in $H_{2}(W)$ which cannot be represented by a smoothly embedded 2-sphere.

Proof. First consider the case where $W=W_{L}$ for some framed link $L$. By Remark 1.11, the class $\alpha$ given by Proposition 6.11 is characteristic for some almost framing on $M_{L}$. By Theorem 3.1, $M_{L}$ with this almost framing bounds a framed manifold $\bar{W}$. The image of $\alpha$ in the second homology of $W \cup \bar{W}$ is characteristic and, according to the Kervaire-Milnor Theorem [5], cannot be represented by a smooth 2 -sphere. Since the image of $\alpha$ cannot be represented by a smooth 2 -sphere in $W \cup \bar{W}$, certainly $\alpha$ cannot be represented by a smooth 2-sphere in $W$. Note that $\alpha \cdot x=0$ for all $x \in H_{2}\left(W_{L}\right)$.

In the general case, as in the proof of Theorem 6.12, framed surgeries can be performed on $W$ to get a parallelizable 4-manifold associated to some framed link. That is, there exist a framed link $L$ with $W_{L}$ parallelizable and framed embeddings $f: S^{1} \rightarrow W, g_{i}: S^{2} \rightarrow W_{L}, i=1, \ldots, n$, such that $W-$ $\left(\cup_{i=1}^{n} f_{i}\left(S^{1}\right)\right)=W_{L}-\left(\cup_{i=1}^{n} g_{i}\left(S^{2}\right)\right)$. Since the class $\alpha \in H_{2}\left(W_{L}\right)$ which cannot be represented by a smooth 2 -sphere satisfies $\alpha \cdot x=0$ for all $x \in$ $H_{2}\left(W_{L}\right), \alpha$ is in the image of $i_{*}: H_{2}\left(W_{L}\right)-\left(\cup_{i=1}^{n} g_{i}\left(S^{2}\right)\right) \rightarrow H_{2}\left(W_{L}\right)$. Thus, there is a class in $H_{2}\left(W-\left(\cup_{i=1}^{n} f_{i}\left(S^{1}\right)\right)\right)$ which cannot be represented by a smooth 2-sphere. The image of this class in $H_{2}(W)$ cannot be represented by a smooth 2 -sphere since, by general position, any such embedding could be chosen to miss $\cup_{i=1}^{n} g_{i}\left(S^{1}\right)$.

\section{REFERENCES}

1. M. Freedman and K. C. Kirby, A geometric proof of Rohlin's theorem, Proc. Sympos Pure Math., vol. 32, part 2, Amer. Math. Soc., Providence, R. I., 1976.

2. C. McA. Gordon, Knots, homology spheres, and contractible 4-manifolds, Topology 14 (1975), 151-172. 
3. J. Harer, A. Kas and R. Kirby, (to appear).

4. N. Jacobson, Basic algebra. I, Freeman, San Francisco, Calif., 1974.

5. M. A. Kervaire and J. Milnor, On 2-spheres in 4-manifolds, Proc. Nat. Acad. Sci. U.S.A. 47 (1961), 1651-1657.

6. R. C. Kirby, A calculus for framed links, Invent Math. 45 (1978), 35-55.

7. W. B. R. Lickorish, A representation of orientable combinatorial 3-manifolds, Ann. of Math. (2)76 (1962), 531-540.

8. J. Milnor, Differentiable manifolds which are homotopy spheres, (mimeographed) Princeton, 1959.

9. R. A. Robertello, An invariant of knot cobordism, Comm. Pure Appl. Math. 18 (1965), 543-555.

10. D. Sullivan, On the intersection ring of compact 3-manifolds, Topology 14 (1975), 275-278.

Department of Mathematics, University of Kentucky, LeXington, Kentucky 40506 